\title{
A Modernização Agropecuária nas Microrregióes do Estado de Minas Gerais
}

\author{
Sílvio Ferreira Júnior ${ }^{1}$ \\ Antônio José M. S. Baptista ${ }^{2}$ \\ João Eustáquio de Lima $^{3}$
}

Resumo: Este estudo objetivou identificar e caracterizar grupos distintos de microrregiões homogêneas quanto ao grau de modernização agropecuária no Estado de Minas Gerais. Para tanto foram empregadas as técnicas de análise fatorial e de análise de agrupamento, a fim de obter os fatores que refletem o padrão tecnológico e cujas relações permitam formar grupos de microrregiões semelhantes. Foram identificados 10 grupos distintos de microrregiões homogêneas, cada um possuindo combinação distinta dos fatores encontrados e que reflete o seu nível tecnológico. Os grupos encontrados constituem informação preliminar para aplicação localizada de políticas de apoio ao setor, bem como para a tomada de decisão por parte dos agentes da iniciativa privada.

Palavras-chave: modernização; nível tecnológico; análise estatística multivariada.

\footnotetext{
${ }^{1}$ Economista, Mestre em Economia Aplicada e Doutorando em Economia Aplicada (Bolsista do CNPq) - DER/UFV, Viçosa, MG. Email: silviofj@hotmail.com

${ }^{2}$ Economista, Mestre em Economia Aplicada e Doutorando em Economia Aplicada (Bolsista PEC-PG/CAPES) - DER/UFV, Viçosa, MG. Email: tozecv@hotmail.com

${ }^{3}$ Ph.D. Professor Titular da Universidade Federal de Viçosa. DER/UFV, Viçosa, MG. Email: jelima@mail.ufv.br
} 


\title{
Cattle Breeding and Agriculture Modernization in Minas Gerais State Microregions
}

\begin{abstract}
The purpose of this study was to identify and characterize different groups of homogeneous microregions concerning cattle breeding and agriculture modernization in Minas Gerais State. In order to achieve it, the factorial analysis techniques and the grouping analysis were used to obtain the factors reflecting the technological standard, factors whose relations enable to organize groups of similar microregions. Ten different groups of homogeneous microregions were identified, and each of them has its own combination of such factors, reflecting its technological level. The groups found in the study constitute preliminary information for localized application of support policies to the sector, as well as for decisions to be made by private initiative agents.
\end{abstract}

Key-words: modernization; technological level; multivaried statistics analysis.

\section{1 - Introdução}

As transformações ocorridas no setor agropecuário brasileiro têm evidenciado, de maneira cada vez mais clara, a íntima relação existente entre modernização agropecuária e o nível tecnológico. Conforme Figueiredo (1996) e Kageyama \& Leone (2002), o processo de modernização está associado a uma produção apoiada no uso combinado e intensivo de insumos modernos, tais como máquinas e tratores, fertilizantes químicos e corretivos, e controle químico de pragas e doenças que resulta em alta produtividade do trabalho e da terra. Adicionalmente, o maior nível tecnológico é acompanhado de mudanças na organização da produção nas relações sociais de trabalho.

De acordo com Kageyama (1996), citado por Souza \& Khan (2001), a modernização da agricultura brasileira pode ser vista a partir de três transformações básicas. Primeira, a modernização tratava, não somente da utilização crescente de insumos modernos, mas também, da mudança das relações de trabalho. A segunda transformação é representada 
pela mecanização, que passou a atuar no processo produtivo desde o plantio até a colheita, substituindo, portanto, a força física e as habilidades manuais do trabalhador, resultando, assim, num salto qualitativo da produção agrícola. A terceira transformação é marcada pela internacionalização dos setores produtivos de insumos, máquinas e equipamentos para a agricultura, a partir da implantação das indústrias de base (siderúrgica, petroquímica, borracha, entre outros) no País, durante as décadas de 50 e 60.

Adotando o mesmo conceito de modernização, Hoffmann (1992) e Figueiredo \& Hoffmann (1998) verificaram, no entanto, que a modernização ocorreu de forma heterogênea, com tecnologias modernas sendo utilizadas nas regiões Sul, Sudeste e em algumas áreas da região Centro-Oeste, enquanto no Norte do País predomina a prática da agricultura tradicional ou mesmo rudimentar.

Este estudo enfocará a agricultura do Estado de Minas Gerais, pela sua importância estratégica na agropecuária do Centro-Sul e pela diferenciação dos programas de desenvolvimento agropecuário aplicados às macrorregiões de planejamento deste estado, e que, pela natureza articulada dos instrumentos de modernização, alguns desses programas serviram como modelo de ação para outros estados brasileiros.

De acordo com Curi (1997), dentre as ações de política de modernização do setor agropecuário mineiro, destacam-se os programas de incorporação de terras baratas do cerrado ao processo produtivo, financiados e comandados pelo poder público durante o período 1960-1980, e a reestruturação do arcabouço institucional responsável pela condução da política agrícola (crédito rural subsidiado para aquisição de máquinas, equipamentos e fertilizantes agrícolas; preços mínimos de garantia; pesquisa e assistência técnica).

A conjugação desses instrumentos contemplou regiões e produtos distintos no território do Estado e contribuiu para acentuar, ainda mais, a heterogeneidade do desenvolvimento agrário, pois levou a uma situação de modernização parcial. Isso porque envolveu, de um lado, a imigração de produtores provenientes de regiões de agricultura mais desenvolvida (Paraná e Rio Grande do Sul), já habituados com técnicas capitalistas de produção e dispostos a se endividarem, medi- 
ante o uso do crédito oficial. Estes produtores se especializaram na produção de commodities (principalmente a soja) e competiram vantajosamente com os produtores da própria região (Noroeste de Minas). E, de outro lado, os produtores mineiros, de perfil oposto ao requerido, que não se enquadravam nos propósitos estabelecidos e que, por isso, deixaram de ser contemplados com os benefícios, ficando à margem do processo de modernização (FUNDAÇÃO JOÃO PINHEIRO, 2000).

Para contrapor à desigualdade no tratamento dispensado, foram criados programas oficiais de estímulo à pequena produção (os Programas de Desenvolvimento Rural Integrado - PDRI). Contudo, esses programas não proporcionaram os resultados esperados a esses agricultores, constituídos basicamente por pequenos e médios proprietários, localizados em regiões diferentes daquelas de cerrado, que permaneceram na produção de bens agropecuários voltados exclusivamente para o mercado interno (arroz, feijão, milho, entre outros), utilizando técnicas pouco evoluídas (Curi, 1997).

Diante da escassez de recursos governamentais, as políticas atuais que visem a modernização da agropecuária e a elevação da sua produtividade devem ser direcionadas às microrregiões mais atrasadas tecnologicamente, de modo a otimizar esses recursos. Qualquer política de modernização da agricultura e da pecuária deve, portanto, considerar as variáveis que definem o padrão tecnológico empregado nas atividades das diferentes microrregiões, bem como detectar semelhanças e diferenças entre estas, a fim de adotar políticas de apoio específicas e coerentes para grupos distintos de microrregiões homogêneas.

Assim sendo, este estudo pretende identificar e caracterizar as microrregiões do Estado de Minas Gerais, quanto ao seu grau de modernização agropecuária, utilizando, para isso, um método de agrupamento, baseado em um conjunto de variáveis que caracteriza o padrão tecnológico e cujas relações permitam definir grupos homogêneos de microrregiões. Especificamente, pretende-se estratificar as microrregiões em grupos homogêneos, identificar as variáveis mais relevantes para cada grupo estratificado e mensurar e hierarquizar cada grupo quanto ao grau de modernização agropecuária. 


\section{2 - Método}

Para o presente estudo, dado o caráter multidimensional do conceito de modernização agropecuária, utilizaram-se as técnicas da análise estatística multivariada, especificamente, a análise fatorial e a análise de agrupamento.

\subsection{Análise fatorial}

A análise fatorial tem como princípio básico a redução do número original de variáveis, por meio da extração de fatores independentes, de tal forma que estes fatores possam explicar, de forma simples e reduzida, as variáveis originais ${ }^{4}$.

A análise fatorial geralmente se realiza através do método de componentes principais, que faz com que o primeiro fator contenha o maior percentual de explicação da variância total das variáveis da amostra. O segundo fator, por sua vez, contenha o segundo maior percentual, e assim por diante.

Cada fator, portanto, consiste em uma combinação linear das variáveis originais padronizadas incluídas no estudo. Na composição destes fatores, têm-se os seguintes princípios: as variáveis mais correlacionadas combinam-se dentro de um mesmo fator; as variáveis que compõem um fator são praticamente independentes das que compõem outros fatores; a derivação dos fatores processa-se, visando maximizar a percentagem de variância total relativo a cada fator consecutivo; e os fatores não são correlacionados entre si. Procura-se, assim, determinar os coeficientes que relacionam as variáveis observadas com os fatores comuns. Esses coeficientes denominados de cargas fatoriais desempenham a mesma função dos coeficientes de correlação.

Para facilitar a interpretação dos fatores é realizada uma rotação ortogonal pelo método Varimax, que procura minimizar o número de variáveis fortemente relacionadas com cada fator, permitindo, assim, obter fatores mais facilmente interpretáveis.

\footnotetext{
${ }^{4}$ Informações detalhadas sobre a análise fatorial podem ser encontradas em Kim \& Muelher (1979) ou Manly (1986).
} 
O modelo de análise fatorial pode ser expressa algebricamente da seguinte forma:

$$
X_{i}=a_{i 1} F_{1}+a_{i 2} F_{2}+\ldots+a_{i m} F_{m}+e_{i}
$$

em que $\mathrm{X}_{\mathrm{i}}$ representa o i-ésimo escore da variável padronizada, com média zero e variância unitária $(i=1,2, \ldots \mathrm{m}) ; \mathrm{F}_{j}$ indica os fatores comuns não correlacionados, com média zero e variância unitária; $a_{i j}$ representa as cargas fatoriais, e $e_{i}$, o termo de erro que capta a variação especifica de $\mathrm{X}_{\mathrm{i}}$ não explicada pela combinação linear das cargas fatoriais com os fatores comuns.

O quadrado das cargas fatoriais representa a contribuição relativa de cada fator para a variância total de uma variável. A soma dessas cargas fatoriais ao quadrado, para cada variável, oferece a estimativa da comunalidade, que, por sua vez, indica a proporção da variância total de cada variável que é explicada pelo conjunto de fatores comuns.

Após calcular as cargas fatoriais e identificar os fatores, torna-se necessário a estimação do escore fatorial, por meio do método semelhante ao de regressão. O escore para cada observação (microrregião), é, portanto, resultado da multiplicação do valor (padronizado) das variáveis pelo coeficiente do escore fatorial correspondente, sendo a expressão geral para estimação do j-ésimo fator, $F_{j}$, dada por:

$$
F_{j}=W_{j 1} X_{1}+W_{j 2} X_{2}+W_{j 3} X_{3}+\ldots+W_{j p} X_{p}
$$

em que os $W_{j i}$ são os coeficientes dos escores fatoriais e $p$ é o número de variáveis.

Para testar a adequabilidade do modelo de análise fatorial, geralmente utiliza-se a estatística de Kaiser-Meyer-Olkin (KMO) e o teste de Bartlett. O KMO é um indicador que compara a magnitude do coeficiente de correlação observado com a magnitude do coeficiente de correlação parcial. Levando em conta que os valores deste teste variam de 0 a 1, pequenos valores de KMO (abaixo de 0,50) indicam a não adequabilidade da análise. Por sua vez, o teste de esfericidade de Bartlett serve para testar a hipótese nula de que a matriz de correlação é uma matriz identidade. Se esta hipótese for rejeitada a análise pode ser realizada. 
Na determinação do número de fatores necessários para representar o conjunto de dados, deve-se considerar a sua contribuição individual e adicional para a variância "explicada". Usualmente, consideramse apenas os fatores cuja raiz característica é maior do que a unidade, isto é, aqueles que correspondem a uma proporção da variância superior àquela atribuída a uma variável isolada. No entanto, não há critério definitivo para essa determinação, sendo isso uma tarefa dos pesquisadores, que baseiam sua decisão na análise do significado descritivo dos fatores.

Após a determinação e a interpretação dos fatores, é possível, finalmente, calcular os escores fatoriais para cada uma das microrregiões do Estado. Estes escores serão utilizados para identificar e agrupar as microrregiões em grupos homogêneos, por meio da análise de agrupamento.

\section{2 - Análise de agrupamento}

A técnica de análise de agrupamento tem o propósito de identificar e classificar unidades (variáveis ou objetos) em grupos distintos, de acordo com determinadas características, a partir de indicadores de semelhança (Manly, 1986). No presente estudo, realizou-se a análise de agrupamento de objetos (microrregiões).

A análise de agrupamento utiliza o conceito de distância entre as unidades de classificação. Há diversos métodos para mensuração dessa distância, dentre os quais o mais utilizado é a distância euclidiana. Assim, são selecionadas as variáveis a serem padronizadas, e, em seguida, é construída uma matriz de distância euclidiana para o processo de agrupamento dos objetos. Esta distância é expressa algebricamente por:

$$
D_{A B}=\sqrt{\sum_{i=1}^{m}\left(X_{A j}-X_{B j}\right)^{2}}
$$

em que, $D_{A B}$ é a medida de distância euclidiana do objeto A ao B; j é o indexador das variáveis. Quanto mais próxima de zero for a distância, maior a similaridade entre os objetos em comparação. 
Há diversos métodos disponíveis para combinação dos objetos em grupos, os quais são classificados como hierárquicos e não-hierárquicos. Os métodos hierárquicos podem ser aglomerativos ou divisivos. Neste estudo utilizou-se o método seqüencial, aglomerativo e hierárquico.

Para a determinação do número de grupos a serem considerados, não há critério pré-estabelecido, sendo necessária a avaliação crítica dos pesquisadores em cada caso específico.

\section{3 - Natureza e fonte dos dados}

Dado o caráter multidimensional da modernização da agropecuária, torna-se necessário um grande número de indicadores para caracterizála. Assim, as variáveis utilizadas neste estudo buscam caracterizar a modernização na agricultura, levando em conta que esta se manifesta por meio da produtividade da terra, da produtividade do trabalho e também se reflete na intensidade do uso de tecnologias modernas (máquinas, uso de adubos químicos, fertilizantes, irrigação, controle de pragas, assistência técnica etc.).

Foram selecionados 22 indicadores de modernização, calculados a partir dos dados disponíveis no Censo Agropecuário do Estado de Minas Gerais, 95/96, publicado pelo IBGE. Tais indicadores são apresentados em termos proporcionais à: área explorada (AE), equivalente homem (EH) e total de estabelecimentos (TE) e foram selecionados de acordo com a relevância para diferenciar e agrupar as microrregiões quanto ao grau de modernização ${ }^{5}$.

O conceito de área explorada (AE) refere-se à soma das áreas com lavouras permanentes e temporárias, pastagens plantadas, matas plantadas, áreas com pastagens naturais e matas naturais.

$\mathrm{O}$ equivalente-homem (EH) refere-se à homogeneização do trabalho de homens, mulheres e crianças, de acordo com a metodologia proposta por Silva \& Kageyama (1983).

Os indicadores de modernização utilizados no estudo foram: X1 número de tratores /AE; X2 - número de tratores /EH; X3 - número de

\footnotetext{
${ }^{5}$ Hoffmann (1992), Figueiredo \& Hoffmann, (1998) e Souza \& Khan (2001), utilizaram, em seus estudos, variáveis semelhantes com intuito de caracterizar o grau de modernização entre regiões diferentes.
} 
estabelecimento com controle de pragas e doenças / AE; X4 - número de estabelecimento que aplica adubos e corretivos / TE; X5 - número de estabelecimento com eletricidade / TE; X6 - quantidade de eletricidade consumida /AE; X7 - área irrigada /AE; X8 - quantidade consumida de combustíveis e lubrificantes /AE; X9 - EH / AE; X10 número de estabelecimento com assistência técnica / AE; X11 - valor de financiamentos /AE; X12 - valor de investimentos /AE; X13 - valor de financiamentos / EH; X14 - valor de investimentos / EH; X15 - valor da produção /AE; X16 - valor da produção / EH; X17 - despesas /AE; $\mathbf{X 1 8}$ - despesas / EH; X19 - número de estabelecimento com controle de pragas e doenças / EH; $\mathbf{X 2 0}$ - número de estabelecimento com assistência técnica / EH; X21 - área irrigada / EH; e X22 - área irrigada / TE.

Estes indicadores foram utilizados no processo de análise fatorial com o intuito de sintetizar algumas medidas do grau de modernização na agropecuária das microrregiões do Estado de Minas Gerais. De posse dos escores fatoriais, referentes a cada uma das 66 microrregiões do Estado de Minas Gerais, procedeu-se à análise de agrupamento, com o intuito de identificar e agrupar microrregiões homogêneas em termos do grau de modernização.

Para operacionalizar a análise, foram utilizados os Softwares SPSS, versão 10 e STATISTICA, versão 5.5.

\section{3 - Resultados e discussão}

\section{1 - Determinação do número de fatores}

Conforme o Quadro 1, foram obtidos, após rotação ortogonal, 3 fatores com raiz característica maior que a unidade e que sintetizam as informações contidas nas 22 variáveis originais. Percebe-se que os fatores F1, F2, e F3 “explicam”, respectivamente, 37,52\%, 28,63\%, 12,85\% da variância total das variáveis utilizadas, e que, em conjunto, explicam $79 \%$.

O teste de Bartlett mostrou-se significativo a $1 \%$, rejeitando a hipótese nula de que a matriz de correlação é uma matriz identidade. O teste de KMO, para a análise da adequabilidade da amostra apresentou um valor de 0,65 , indicando que a amostra é passível de ser analisada pelas técnicas da análise fatorial. 
Quadro 1 - Raiz característica e percentual explicado por cada fator

\begin{tabular}{cccc}
\hline Fator & Raiz característica & $\begin{array}{c}\text { Variância explicada } \\
\text { pelo fator (\%) }\end{array}$ & $\begin{array}{c}\text { Variância acumulada } \\
(\%)\end{array}$ \\
\hline F1 & 8,255 & 37,52 & 37,52 \\
F2 & 6,300 & 28,63 & 66,16 \\
F3 & 2,827 & 12,85 & 79,00 \\
\hline \multicolumn{2}{r}{ Teste de esfericidade de Bartlett $=2.572,01(\mathrm{p}<1 \%)$ e KMO $=0,651}$.
\end{tabular}

O Quadro 2 apresenta as cargas fatoriais e as comunalidades para os 3 fatores considerados. Para a interpretação de cada um dos fatores, foram consideradas apenas as cargas fatoriais com valores superiores a 0,50 (destacadas em negrito). Os valores encontrados para as comunalidades revelam que praticamente todas as variáveis têm a sua variabilidade significativamente captada e representada pelos 3 fatores.

Quadro 2- Cargas fatoriais após rotação ortogonal e as comunalidades

\begin{tabular}{lcccc}
\hline \multicolumn{1}{c}{ Indicadores } & F1 & F2 & F3 & Comunalidades \\
\hline X1 (tratores/AE) & $\mathbf{0 , 8 3 9}$ & 0,260 & 0,009 & 0,772 \\
X2 (tratores/EH) & 0,191 & $\mathbf{0 , 9 2 1}$ & $-0,011$ & 0,886 \\
X3 (p\&d/AE) & $\mathbf{0 , 7 8 1}$ & $-0,448$ & 0,064 & 0,814 \\
X4 (a\&c/TE) & $\mathbf{0 , 7 2 8}$ & 0,273 & 0,107 & 0,616 \\
X5 (eletric./TE) & $\mathbf{0 , 6 2 1}$ & 0,458 & 0,172 & 0,625 \\
X6 (eletric/AE) & $\mathbf{0 , 8 7 6}$ & 0,029 & $-0,013$ & 0,768 \\
X7 (área irrig/AE) & 0,247 & $-0,151$ & $\mathbf{0 , 8 7 4}$ & 0,848 \\
X8 (comb\&Lub/AE) & $\mathbf{0 , 8 3 0}$ & 0,397 & $-0,037$ & 0,848 \\
X9 (EH/AE) & $\mathbf{0 , 7 0 9}$ & $-0,592$ & $-0,064$ & 0,857 \\
X10 (as.téc./AE) & $\mathbf{0 , 9 2 4}$ & 0,038 & 0,084 & 0,861 \\
X11 (financ/AE) & $\mathbf{0 , 5 7 4}$ & 0,383 & $-0,206$ & 0,519 \\
X12 (inv/AE) & $\mathbf{0 , 8 7 4}$ & 0,291 & 0,012 & 0,849 \\
X13 (financ/EH) & 0,011 & $\mathbf{0 , 8 1 4}$ & $-0,179$ & 0,694 \\
X14 (inv/EH) & $-0,017$ & $\mathbf{0 , 9 1 9}$ & 0,040 & 0,847 \\
X15 (v.produção/AE) & $\mathbf{0 , 9 4 1}$ & 0,146 & $-0,027$ & 0,908 \\
X16 (v.produção/EH) & 0,249 & $\mathbf{0 , 9 2 1}$ & $-0,002$ & 0,910 \\
X17 (despesa/AE) & $\mathbf{0 , 8 8 9}$ & 0,211 & $-0,013$ & 0,834 \\
X18 (despesa/EH) & 0,262 & $\mathbf{0 , 8 8 9}$ & 0,015 & 0,859 \\
X19 (p\&d/EH) & 0,247 & $\mathbf{0 , 5 2 0}$ & 0,323 & 0,435 \\
X20 (as.téc./EH) & 0,361 & $\mathbf{0 , 7 9 9}$ & 0,106 & 0,779 \\
X21 (área irrig/EH) & $-0,086$ & 0,090 & $\mathbf{0 , 9 6 8}$ & 0,952 \\
X22 (área irrig/TE) & $-0,136$ & 0,074 & $\mathbf{0 , 9 3 5}$ & 0,899 \\
\hline
\end{tabular}

Fonte: Resultados da pesquisa. 
Percebe-se que o fator F1 está positiva e fortemente relacionado com os indicadores X4 e X5, que expressam as variáveis em relação ao total de estabelecimentos, e com os indicadores X1, X3, X6, X8, X9, $\mathrm{X} 10, \mathrm{X} 11, \mathrm{X} 12, \mathrm{X} 15$ e X17, que expressam as variáveis em relação à área explorada. De certa forma, estes indicadores estão relacionados com a intensidade do uso da terra. Assim, a natureza dos indicadores que se relacionam com F1 indica que este representa a "intensidade do uso da terra”.

$\mathrm{O}$ fator F2, por sua vez, é positiva e fortemente relacionado com os indicadores X2, X13, X14, X16, X18, X19 e X20, que expressam as variáveis em relação ao equivalente-homem. Optou-se, assim, por denomina-lo "relação capital/trabalho".

$\mathrm{O}$ fator F3, por sua vez, está positiva e fortemente relacionado com os indicadores X7, X21, X22, que expressam a área irrigada em relação à área total, ao equivalente-homem e ao total de estabelecimentos, respectivamente. Assim, convencionou-se denominar F3 de "área irrigada”.

De posse das cargas fatoriais, o passo seguinte foi determinar os escores fatoriais, ou seja, determinar o valor dos fatores para cada microrregião do Estado de Minas Gerais. Esses escores serão utilizados para agrupar as microrregiões em grupos homogêneos.

\section{2 - Determinação do número de agrupamentos}

Durante o estudo dos resultados da análise de agrupamento, constatou-se elevado grau de desigualdade no que se refere aos níveis tecnológicos encontrados em cada microrregião do Estado de Minas Gerais, implicando maior dificuldade para formar agrupamentos com significativo grau de homogeneidade interna. Portanto, a fim de preservar a característica própria dos agrupamentos considerados, trabalhouse com maior número destes, considerando o total de 10 grupos distintos de microrregiões homogêneas.

Após a formação dos grupos, foram calculados os escores fatoriais médios e o nível tecnológico de cada grupo, conforme o Quadro 3. Cada escore médio (colunas 2, 3 e 4) foi obtido por meio da soma do escore de cada microrregião, ponderado pela sua participação no valor total da produção agropecuária no grupo em que ela se insere. 
O nível tecnológico, ou o grau de modernização para cada grupo, refere-se à média aritmética dos escores médios encontrados, que produz um índice bruto (coluna 5). De posse dessa informação, construiuse um índice relativo na base 100 , de forma que o maior valor se tornou igual a 100 e o menor valor igual a zero, com os valores intermediários sendo alocados por meio de interpolação (coluna 6). Assim, obteve-se a posição relativa dos grupos formados no que se refere ao nível tecnológico empregado na agropecuária ${ }^{6}$.

A análise dos fatores F1, F2 e F3 deve ser feita levando em conta que seus escores originais, quando consideradas todas as microrregiões em conjunto, são variáveis com média zero e desvio padrão igual a 1. Portanto, pode-se interpretar que os escores com valores próximos de zero indicam nível médio de tecnologia, ou, de modernização agropecuária, no que se refere ao significado do fator em consideração. Quanto maior em relação a zero for o escore fatorial, tanto mais avançada tecnologicamente será a agropecuária do grupo em análise.

De posse dessas informações, podem ser verificadas as diferenças quanto ao grau de modernização entre os grupos formados, conforme o Quadro 3.

O grupo 1 refere-se à microrregião de Formiga, isoladamente, que responde por 1,14\% do valor da produção agropecuária do Estado. Essa microrregião apresentou valores positivos em todos os três escores. Embora a intensidade do uso da terra (F1) e a relação capital/trabalho (F2) tenham apresentado valores pouco acima da média do Estado, o fator que representa a área irrigada (F3) destacou-se por apresentar-se bem acima da média e foi decisivo para classificar essa microrregião como a de maior nível tecnológico, da ordem de 1,58, bem acima, portanto, da média estadual. Essa microrregião, portanto, recebeu índice tecnológico igual a 100.

\footnotetext{
${ }^{6}$ Souza \& Khan (2001) utilizaram essa mesma técnica para a hierarquização dos municípios maranhenses quanto ao nível tecnológico empregado na agricultura.
} 
Quadro 3 - Classificação relativa em ordem decrescente do nível tecnológico e participação percentual no valor bruto da produção agropecuária para os 10 grupos de microrregiões homogêneas do Estado de Minas Gerais

\begin{tabular}{rrrrccc}
\hline Grupo & F1 & F2 & F3 & $\begin{array}{c}\text { Índice } \\
\text { bruto }\end{array}$ & $\begin{array}{c}\text { Índice na } \\
\text { base } 100\end{array}$ & $\begin{array}{c}\% \text { do VBP } \\
\text { do Estado }\end{array}$ \\
\hline 1 & 0,227 & 0,112 & 4,400 & 1,58 & 100,00 & 1,14 \\
2 & $-0,615$ & 1,502 & 1,228 & 0,70 & 61,22 & 5,76 \\
3 & 1,402 & $-1,171$ & 1,806 & 0,68 & 60,07 & 2,99 \\
4 & $-0,297$ & 2,198 & $-0,431$ & 0,49 & 51,70 & 17,24 \\
5 & 1,334 & 0,146 & $-0,439$ & 0,35 & 45,38 & 24,65 \\
6 & $-0,611$ & $-0,509$ & 1,651 & 0,18 & 37,83 & 2,01 \\
7 & 0,088 & $-0,181$ & 0,601 & 0,17 & 37,49 & 7,62 \\
8 & $-0,490$ & 0,902 & $-0,447$ & $-0,01$ & 29,47 & 14,09 \\
9 & 0,636 & $-1,067$ & $-0,541$ & $-0,32$ & 15,63 & 9,84 \\
10 & $-0,919$ & $-0,654$ & $-0,458$ & $-0,68$ & 0,00 & 14,68 \\
\hline
\end{tabular}

Fonte: Resultados da pesquisa.

No grupo 2 estão inseridas as microrregiões de Frutal, Três Marias e Piuí, em ordem decrescente do nível tecnológico, que respondem, juntas, por 5,76\% do valor da produção agropecuária do Estado. Percebese que os fatores médios deste grupo, no que se refere à relação capital/ trabalho (F2) e à área irrigada (F3), apresentaram valores bem acima da média estadual. No entanto, o fator que representa a intensidade do uso da terra apresentou valor abaixo da média estadual, o que determinou, juntamente com os demais fatores, a ocupação desse grupo na segunda colocação quanto ao nível tecnológico, que foi 38,7\% menor que o nível verificado no grupo 1 (Quadro 3, última coluna).

No grupo 3, estão inseridas as microrregiões de Poços de Caldas e Itaguara, em ordem decrescente do nível tecnológico, que respondem, juntas, por 2,99\% do valor da produção agropecuária do Estado. Observa-se que esse grupo caracterizou-se por apresentar valores positivos e elevados nos fatores que representam a intensidade do uso da terra (F1) e a área irrigada (F3). No entanto, o fator que representa a relação capital/trabalho revelou-se bem abaixo da média estadual, de modo que este grupo apresentou nível tecnológico aproximadamente $40 \%$ inferior ao nível verificado no grupo 1, ocupando a terceira colocação geral na classificação obtida.

No grupo 4, estão inseridas as microrregiões de Patrocínio, Uberaba, 
Uberlândia, Ituiutaba e Araxá, em ordem decrescente do nível tecnológico, e que representam $7,6 \%$ das microrregiões e $17,24 \%$ do valor da produção agropecuária do Estado. Embora tenha apresentado valores abaixo da média estadual, no que se refere aos fatores F1 e F3, esse grupo se destacou por ter apresentado valor bem acima da média no que se refere à relação capital/trabalho (F2). Esse grupo apresentou nível tecnológico 48,3\% menor que o verificado no grupo 1 e obteve a quarta colocação geral.

No grupo 5, estão inseridas as microrregiões de Pará de Minas, Varginha, Alfenas, São Sebastião do Paraíso, Santa Rita do Sapucaí, Passos, Lavras, Belo Horizonte, Pouso Alegre, Campo Belo, Barbacena e São Lourenço, que representam $18,2 \%$ das microrregiões e $24,65 \%$ do valor da produção agropecuária do Estado. Este grupo caracterizou-se por ter apresentado valor alto e positivo no fator F1, valor baixo e positivo no fator F2 e valor baixo e negativo no fator F3, determinando sua ocupação na quinta colocação quanto ao nível tecnológico, que foi $54,62 \%$ menor que o verificado no grupo 1 .

No grupo 6 estão inseridas as microrregiões de Ipatinga, Ouro Preto, Pirapora e Guanhães, que representam 6,1\% das microrregiões e 2,01 \% do valor da produção agropecuária do Estado. Este grupo caracterizou-se por ter apresentado valores abaixo da média estadual nos escores F1 e F2 e valor positivo e elevado no escore F2, determinando sua ocupação na sexta colocação quanto ao nível tecnológico, que foi $62,17 \%$ inferior ao nível verificado no grupo 1 .

No grupo 7 estão inseridas as microrregiões de Divinópolis, Oliveira, Itabira, São João Del Rei, Itajubá, Cataguases e Andrelândia, que representam 10,6\% das microrregiões e 7,62 \% do valor da produção agropecuária do Estado. Este grupo caracterizou-se por ter apresentado valores pouco acima da média nos escores F1 e F3 e valor pouco abaixo da média no escore F3, determinando sua ocupação na sétima colocação quanto ao nível tecnológico, que foi 62,51\% inferior ao nível verificado no grupo 1 .

No grupo 8 estão inseridas as microrregiões de Patos de Minas, Bom Despacho, Paracatu, Sete Lagoas, Unaí e Curvelo, que representam 9,1\% das microrregiões e 14,09\% do valor da produção agropecuária do Estado. Este grupo caracterizou-se por ter apresentado valores abaixo da média nos fatores F1 e F3 e valor acima da média no fator F2. Este grupo carac- 
terizou-se, ainda, por inserir as microrregiões que apresentaram nível tecnológico bem próximo da média do Estado (índice bruto próximo de zero), determinando sua ocupação na oitava colocação quanto ao nível tecnológico, que foi 70,53\% inferior ao nível verificado no grupo 1 .

No grupo 9 estão inseridas as microrregiões de Ubá, Manhuaçú, Conselheiro Lafaiete, Muriaé, Viçosa, Ponte Nova e Caratinga, que representam 10,6\% das microrregiões e 9,84\% do valor da produção agropecuária do Estado. Este grupo caracterizou-se por ter apresentado valores abaixo da média nos fatores F2 e F3 e valor acima da média no fator F1, determinando sua ocupação na nona colocação quanto ao nível tecnológico, que foi $84,37 \%$ inferior ao nível verificado no grupo 1 .

Por fim, no grupo 10 estão inseridas as microrregiões de Manuque, Juiz de Fora, Pedra Azul, Aimorés, Conceição do Mato Dentro, Governador Valadares, Janaúba, Mantena, Diamantina, Capelinha, Peçanha, Montes Claros, Macaiúva, Salinas, Teófilo Otoni, Almenara, Januária, Araçuaí e Grão Mogol, que representam 28,8\% das microrregiões e $14,68 \%$ do valor da produção agropecuária do Estado. Este grupo caracterizou-se por ter apresentado valores bem abaixo da média nos três escores considerados, determinando sua ocupação na última colocação quanto ao nível tecnológico, que foi $100 \%$ inferior ao nível verificado no grupo 1. Adicionalmente, esse grupo apresentou os menores valores médios nos indicadores X1, X4, X5, X6, X8, X10, X15, X16, X17, $\mathrm{X} 18, \mathrm{X} 20$ e X23, quando comparados aos demais grupos. Isso, certamente, em associação com os demais indicadores, contribuiu para a última posição relativa desse grupo na classificação obtida.

\section{4 - Conclusões}

A análise fatorial conseguiu reduzir o grande número de indicadores a um pequeno número de fatores que sintetizam o caráter multidimensional da modernização da agropecuária. Os escores fatoriais computados para cada microrregião, possibilitaram a distinção de 10 grupos distintos, em função do nível tecnológico ou grau de modernização da agropecuária no Estado de Minas Gerais.

Os resultados revelam diferenças significativas entre os grupos formados. Três grupos apresentaram nível tecnológico abaixo da média 
estadual, representando, juntas, 48,5\% das microrregiões e $38,6 \%$ do valor da produção agropecuária do Estado. Seis grupos apresentaram nível tecnológico inferior a $50 \%$ do nível verificado no grupo mais modernizado, representando, juntos, 83,4\% das microrregiões e 73,9\% do valor da produção agropecuária do Estado. Ressalta-se, mais uma vez, que a classificação obtida para cada grupo representa sua posição relativa aos demais, dentro do Estado de Minas Gerais.

É importante considerar que, se por um lado, maior nível tecnológico proporciona maior produtividade, por outro lado, o aumento desta também pode ser obtido por meio da melhor utilização dos insumos (melhora na eficiência), mantido constante o nível tecnológico. Assim, a ordem na classificação dos grupos e das microrregiões quanto ao nível tecnológico relativo, não necessariamente seria a mesma, caso as variáveis consideradas fossem a eficiência técnica e/ou a produtividade dos insumos utilizados na produção.

Por conseguinte, os resultados deste estudo não esgotam os debates sobre o tema, mas revelam-se importantes na medida em que fornecem subsídios adicionais que podem contribuir para elaboração de políticas agrícolas mais coerentes com as especificidades existentes em cada grupo de microrregiões do Estado de Minas Gerais, aumentando, assim, as chances de sucesso.

\section{5 - Referências Bibliográficas}

CURI, WALTER FOUAD. Eficiência e fonte de crescimento da agricultura mineira na dinâmica de ajustamentos da economia brasileira. Viçosa, MG: UFV, Imprensa Universitária, 1997. 110 f. Dissertação (Mestrado em Economia Rural) - Universidade Federal de Viçosa.

FIGUEIREDO, N. M. S. Modernização, distribuição da renda e pobreza na agricultura brasileira, 1975, 1980 e 1985. Piracicaba, SP: ESALQ, 1996. 248 f. Tese (Doutorado) - Escola Superior de Agricultura "Luis de Queiroz", Universidade de São Paulo.

FIGUEIREDO, N. M. S.; HOFFMANN, R.. A dinâmica da modernização da agricultura em 299 microrregiões homogêneas do Brasil: 1975, 1980 e 1985. In CONGRESSO BRASILEIRO DE ECONOMIA E SOCIOLOGIA RURAL, 36, 1998, Poços de Caldas. Anais... Poços de Caldas: SOBER, 1998. p. 439-450. 
FUNDAÇÃO JOÃO PINHEIRO. Perfil de Minas Gerais. Belo horizonte: Fundação João Pinheiro, 2000.

HOFFMANN, R. A dinâmica da modernização da agricultura em 157 microrregiões homogêneas do Brasil. Revista de Economia e Sociologia Rural, Brasília, v.30, n.4, p. 271-290. 1992.

KAGEYAMA, A. \& LEONE E. Trajetórias da modernização e emprego agrícola no Brasil, 1985-1996. Revista de Economia e Sociologia Rural, Brasília, v.40, n. 1, p. 9-28. 2002.

KIM, J.O; MUELHER, C.W. Introduction to factor analysis: What it is and how to do it. Beverly Hills: SAGE, 1978. 79 p. (Series quantitative Applications in the Social Science, 7-13).

MANLY, B.F.J. Multivariate statistical methods - a primer. New York: Chapman and Hall, 1986. 159 p.

SILVA, J.G; KAGEYAMA, A.A. Emprego e relações de trabalho na agricultura Brasileira: Uma análise dos dados censitários de 1960, 1970, 1975. Pesquisa e Planejamento Econômico. 13(1). Abr. 1983.

SOUZA, R. F.; A. S. KHAN. Modernização da agricultura e hierarquização dos municípios maranhenses. Revista de Economia e Sociologia Rural. Brasília, v.39, n.2, p. 81-104, 2001.

Recebido em abril de 2003 e revisto em junho de 2003. 
